kcl-th-93-4

March 1993

\title{
A Covariant Canonical Description of Liouville Field Theory
}

\author{
G. PAPAdopoulos \\ Department of Mathematics \\ King's College London \\ London WC2R 2LS \\ and \\ B. SPENCE \\ School of Physics \\ University of Melbourne \\ Parkville 3052 Australia
}

\begin{abstract}
We present a new parameterisation of the space of solutions of Liouville field theory on a cylinder. In this parameterisation, the solutions are well-defined and manifestly real functions over all space-time and all of parameter space. We show that the resulting covariant phase space of the Liouville theory is diffeomorphic to the Hamiltonian one, and to the space of initial data of the theory. The Poisson brackets are derived in our approach, and shown to be those of the co-tangent bundle of the loop group of the real line. Using Hamiltonian reduction, we show that our covariant phase space formulation of Liouville theory may also be obtained from the covariant phase space formulation of the Wess-Zumino-Witten model that we have given previously.
\end{abstract}




\section{Introduction}

The Liouville model [1] is a venerable field theory, the study of which has absorbed mathematicians and physicists for more than a century. This theory has many applications in conformal field theory, $2 \mathrm{~d}$ gravity and particularily in the theory of non-critical strings [2]. Liouville theory has a number of interesting classical and quantum properties. For example, there are Bäcklund transformations that relate the Liouville theory to a free field theory [3-6] and a quantum group structure arises in this theory [7-12]. The study of these properties utilises an explicit parameterisation of the solutions of the classical field equations of Liouville theory, and the calculation of the Poisson brackets of the parameters of these solutions.

The solutions $\phi$ of Liouville theory are functions from a 1+1-dimensional spacetime into the real numbers $\mathcal{R}$ and they are parameterised by two independent parameters per space-time point. In the literature there are two distinct parameterisations of the solutions of Liouville theory [3-5, 13-15]. In the first, the solutions are parameterised by two real (or complex) independent functions $\{A, B\}$ of the light-cone co-ordinates $(x \lambda+, x \lambda-) \equiv(x+t, x-t)$ respectively. In addition, for the Liouville theory on a cylinder an $S L(2, \mathcal{R})$ (or $S L(2, \mathcal{C})$ ) monodromy $M$ can be introduced that acts non-linearly on the parameters $\{A, B\}$. In the second approach, the space of solutions of Liouville theory is parameterised by four real (or complex) functions $\{u \lambda i, v \lambda i\}, i=1,2$. Both $u$ and $v$ transform under the fundamental representation of $S L(2, \mathcal{R})$ (or $S L(2, \mathcal{C})$ ) and depend on the lightcone co-ordinates $(x \lambda+, x \lambda-)$ respectively. However, in this case two $S L(2, \mathcal{R})$ (or $S L(2, \mathcal{C})$ )-invariant constraints are introduced that depend on $\{u, v\}$ and their first derivatives. This reduces the number of independent parameters to two. In this case the monodromy $M$ acts linearly on $u$ and $v$. The two distinct ways of parameterising the space of solutions of Liouville theory can be related by constructing a map from the $\{u, v\}$ to the $\{A, B\}$ parameters [13].

In both of these parameterisations of the space of solutions of Liouville theory, 
the relationship between the parameters of the space of solutions and the initial data of the theory is implicit (for a discussion of this point see ref. [13]). To our knowledge, no (explicit) diffeomorphism has been constructed which relates the parameters of the solutions to the initial data of the Liouville theory. Another problem that arises in the above parameterisations is that for certain values of the parameters the solutions of the Liouville theory become complex; this is the case even if the parameters of the solutions are real functions. The reality condition on the solutions imposes restrictions on their parameters. These restrictions are nonlinear in the parameters and their first derivatives, and they affect the quantum mechanical treatment of the theory. Furthermore, the solutions of the Liouville theory expressed in the above parameterisations are not well-defined on some regions of space-time for certain choices of parameters. (For example, in the $\{A, B\}$ parameterisation, the Liouville solution $e \lambda-2 \lambda \phi=\left(\partial_{+} A \partial_{-} B\right) /(1-\eta \lambda 2 A B) \lambda 2(\lambda$ and $\eta$ are coupling constants) is not defined at $t=0$ for $A=\eta \lambda 2 / B$.) Similar problems also appear when the solutions of the Liouville theory are expressed in terms of a free field and they have been discussed by the authors of refs. [4]. The above problems are closely related to the difficulty of specifying the ranges of the parameters $\{A, B\}$ and $\{u, v\}$ and their first derivatives that parameterise the solutions of the Liouville theory.

In a recent paper [16], see also [17], we have given a new parameterisation of the space of solutions of the Wess-Zumino-Witten (WZW) model with target space a compact and connected group $G$, and we showed that the covariant and Hamiltonian phase spaces of this theory are diffeomorphic. The key point of this construction was the introduction of a connection as part of the parameterisation of the solutions of the WZW model. We showed how the parameters of our new parameterisation of the WZW solutions are related to the initial data of the theory by a simple transformation. We finally used this new formulation to calculate the Poisson brackets of the WZW theory in the covariant canonical approach.

In this paper, we will present a new parameterisation of the space of solutions of the Liouville field theory on a cylinder. As in the case of the WZW model, this 
involves the introduction of a connection. We will show that in this parameterisation the solutions of the Liouville theory are well-defined functions from a cylinder (space-time) into the real numbers. We will then derive the Poisson brackets of the theory and construct a symplectic diffeomorphism that relates the covariant phase space of the Liouville theory to the Hamiltonian one, and to the space of initial data of the theory. Then we will show how these results can also be derived from the Hamiltonian reduction of the covariant phase space of the WZW model which we have given in ref. [16].

\section{Covariant Canonical Description of Liouville Theory}

The phase space of a classical system can be defined in two different ways. The first gives the Hamiltonian phase space $P_{H}$, which is defined as the space of positions and momenta of a system, equipped with the standard symplectic form. For the second definition, we begin with the space of fields of a system. Then we introduce the symplectic current $S \lambda \mu=\delta \phi \lambda I \delta\left(\partial L / \partial\left(\partial_{\mu} \phi \lambda I\right)\right)$, where $\phi$ is the field and $L$ is the Lagrangian of the theory. This current is conserved $\left(\partial_{\mu} S \lambda \mu=0\right)$ when the Lagrangian equations of motion of the model are satisfied. The Lagrangian symplectic form $\Omega$ is defined as the integral over a Cauchy surface of the time component of the symplectic current. This two-form is closed and independent of the choice of the Cauchy surface that we have used to define it. This symplectic form can be parameterised in different ways. One way is in terms of the initial data of the theory. In theories for which we know an explicit parameterisation of the space of solutions, the Lagrangian symplectic form can also be parameterised directly in terms of the parameters of the solutions. The covariant phase space $P_{C}$ of a theory is then defined as the space of solutions of the Lagrangian equations of motion of the system, equipped with the Lagrangian symplectic form written in terms of the parameters of the solutions.

The Lagrangian of the Liouville theory is

$$
L=-2\left(\partial_{+} \phi \partial_{-} \phi+\frac{\eta \lambda 2}{\lambda \lambda 2} e \lambda-2 \lambda \phi\right)
$$


where $\phi$ is a map from a cylinder $S \lambda 1 \times \mathcal{R}$ to $\mathcal{R}$, and $\lambda$ and $\eta$ are real coupling constants. The pairs $(x, t): 0 \leq x<1,-\infty<t<\infty$ are the co-ordinates of $S \lambda 1 \times \mathcal{R}$ and $x \lambda \pm=x \pm t, \partial_{ \pm}=\frac{1}{2}\left(\partial_{x} \pm \partial_{t}\right)$. The equations of motion are

$$
\partial_{+} \partial_{-} \phi+\frac{\eta \lambda 2}{\lambda} e \lambda-2 \lambda \phi=0 .
$$

The Lagrangian symplectic form of the model is

$$
\Omega=\int_{0} \lambda 1 d x \delta \phi \partial_{t} \delta \phi
$$

evaluated at $t=0$.

To construct a new parameterisation of the space of solutions of the Liouville theory, we set

$$
e \lambda \lambda \phi(x, t)=\epsilon_{i j} u \lambda i(x \lambda+) W \lambda j_{l}(A ; x \lambda+, x \lambda-) v \lambda l(x \lambda-),
$$

where indices $i, j, l=1,2, \epsilon$ is the $2 \times 2$ anti-symmetric matrix with $\epsilon_{12}=1, u$ and $v$ are periodic functions from the real line $\mathcal{R}$ into $\mathcal{R} \lambda 2$, and $W\left(A ; x \lambda+, x \lambda_{-}\right)=$ $\left\{W \lambda j_{l}(A ; x \lambda+, x \lambda-)\right\}$ is the holonomy of a connection $A$ given by

$$
W(A ; x \lambda+, x \lambda-)=P \exp \int_{x \lambda-} \lambda x \lambda+A(s) d s .
$$

In the above, $A$ is a periodic one-form on the real line, with values into $(\operatorname{LieS} L(2, \mathcal{R})) \lambda * \mathbb{\|}$ the dual of the Lie algebra of $S L(2, \mathcal{R})$. Note that we take $u$ to be a function of $x \lambda+$, and $v$ a function of $x \lambda-$. Finally, the functions $u, v$ are required to satisfy the constraints

$$
\epsilon_{i j} u \lambda i \nabla_{+} u \lambda j=-\nu, \quad \epsilon_{i j} v \lambda i \nabla_{-} v \lambda j=\frac{\eta \lambda 2}{\nu},
$$

where the covariant derivatives are given by

$$
\nabla_{+} u \lambda i=\partial_{+} u \lambda i-A \lambda i_{j} u \lambda j, \quad \nabla_{-} v \lambda i=\partial_{-} v \lambda i-A \lambda i_{j} v \lambda j
$$

and $\nu$ is a real constant $(\nu \neq 0)$. We note that the expression for $\phi$ in equation (4) 
above, and the constraints (6), are invariant under the gauge transformations

$$
\begin{aligned}
& u(x) \rightarrow h \lambda-1(x) u(x), \quad v(x) \rightarrow h \lambda-1(x) v(x), \\
& A(x) \rightarrow-\partial_{x} h(x) h \lambda-1(x)+h(x) A(x) h \lambda-1(x),
\end{aligned}
$$

where $h$ is an element of the loop group $L S L(2, \mathcal{R})$ of $S L(2, \mathcal{R})$.

It can be shown from the periodicity of $u, v$ and $A$ that $\phi$ is periodic in $x$. In addition, if $u, v$ and $A$ satisfy the conditions of eqn. (6), then $\phi$ of eqn. (4) solves the equations of motion of Liouville theory, eqn. (2). However note that $\phi$ in equation (2) is not necessarily a real function. As we will explain later, the reality condition on the solutions $\phi$ imposes additional restrictions on the parameters $u, v, A$.

This method of parameterising the solutions of the Liouville theory is similar to (and was inspired by) the one constructed in ref. [16] for the WZW model. Note, however, that in this case there are the conditions of eqn. (6) together with the requirement that the solutions $\phi$ are real functions. These are additional restrictions on the parameters of the theory. Because of this, we will not treat the above construction as fundamental as we did in the case of the WZW model. Rather, we will consider it as a device to give a parameterisation to the space of solutions of Liouville theory.

In order to express the solutions of the field equations in terms of independent parameters and calculate the symplectic form of the covariant phase space of the Liouville theory, we first have to gauge fix the symmetry generated by the group action of eqn. (8) and then solve the conditions of eqn. (6).

Due to eqn. (6), the parameters $u, v$ are not allowed to be zero. To obtain a non-degenerate symplectic form on $P_{C}$ we must fix the gauge symmetry. We will choose the following gauge: writing $v=\left(\begin{array}{l}v \lambda 1 \\ v \lambda 2\end{array}\right)$, at least one of $v \lambda 1, v \lambda 2$ must be non-zero. If $v \lambda 2 \neq 0$, then we choose $u=u_{0}=\left(\begin{array}{l}1 \\ 0\end{array}\right)$, and $v=v_{0}=k\left(\begin{array}{l}0 \\ 1\end{array}\right)$, where 
$k$ is a map from $S \lambda 1$ into the real line minus the origin $\mathcal{R}-\{0\}$ (i.e. $k \neq 0)$. In fact, we will show later (by comparison with the initial data) that $k$ should take values in the positive real line $\mathcal{R} \lambda+-\{0\}$. If $v \lambda 2=0$ (and so $v \lambda 1 \neq 0$ ), then we choose $u=\left(\begin{array}{l}0 \\ 1\end{array}\right)$, and $v=k\left(\begin{array}{l}1 \\ 0\end{array}\right)$.

We now consider the first gauge fixing, i.e. assume that $v \lambda 2 \neq 0$ (analogous results obtain for the other case). Then we pick the following basis in $(\operatorname{Lie} S L(2, \mathcal{R})) \lambda *:$

$t \lambda \lambda 0=\left\{(t \lambda \lambda 0) \lambda i_{j}\right\}=\left(\begin{array}{cc}1 & 0 \\ 0 & -1\end{array}\right), \quad t \lambda+=\left\{(t \lambda+) \lambda i_{j}\right\}=\left(\begin{array}{ll}0 & 1 \\ 0 & 0\end{array}\right), \quad t \lambda-=\left\{(t \lambda-) \lambda i_{j}\right\}=\left(\begin{array}{ll}0 & 0 \\ 1 & 0\end{array}\right)$

Substituting the gauge conditions into the constraints (6) and solving them in terms of the components of $A=A_{0} t \lambda \lambda 0+A_{+} t \lambda++A_{-} t \lambda-$, we get

$$
A_{-}=\nu, \quad A_{+}=\frac{\eta \lambda 2}{\nu} \frac{1}{k \lambda 2}
$$

Thus, the independent variables after gauge-fixing are $k$ and the component $A_{0}$ of the connection $A$. Substituting the gauge-fixing conditions and the solutions (10) of the conditions (6) into the parameterisation (4), we get a parameterisation of the space of solutions of the Liouville theory in terms of $k$ and $A_{0}$. This is

$$
\begin{aligned}
e \lambda \lambda \phi(x, t) & =\epsilon_{i j} u_{0} \lambda i W \lambda j_{l}(a ; x \lambda+, x \lambda-) v_{0} \lambda l(x \lambda-) \\
& =W \lambda 2_{2}(a ; x \lambda+, x \lambda-) k(x \lambda-)
\end{aligned}
$$

where $a=A_{0} t \lambda \lambda 0+\frac{\eta \lambda 2}{\nu} \frac{1}{k \lambda 2} t \lambda++\nu t \lambda-$. In addition, if we require that $k$ takes values into the positive real line, then the solutions $\phi$ (eqn.(11)) of the Liouville theory parameterised in terms of $A_{0}$ and $k$ are functions from a cylinder into the real line $\mathcal{R}$, i.e. they are well-defined and real at every point of the space time for any choice of the parameters $A_{0}$ and $k$ (we will justify this at the end of this section). The space of parameters of the solutions of the Liouville theory is the co-tangent bundle of the loop group $L(\mathcal{R} \lambda+-\{0\})$ and it is diffeomorphic to the co-tangent bundle of $L \mathcal{R}$. 
The Lagrangian symplectic form (3) parameterised in terms of $k, A_{0}$ is then found to be

$$
\Omega=-\frac{1}{\lambda \lambda 2} \int_{0} \lambda 1 d x\left(2 \delta \log (k) \delta A_{0}+\delta \log (k) \partial_{x} \delta \log (k)\right) .
$$

This symplectic form is not degenerate and can be easily inverted. The Poisson brackets of the Liouville theory are thus found to be

$$
\begin{aligned}
\{k(x), k(y)\} & =0 \\
\left\{k(x), A_{0}(y)\right\} & =-\frac{\lambda \lambda 2}{2} k(x) \delta(x, y), \\
\left\{A_{0}(x), A_{0}(y)\right\} & =\frac{\lambda \lambda 2}{2} \partial_{x} \delta(x, y),
\end{aligned}
$$

where $\delta(x, y)$ is the delta function on a circle. Note that in the variables $q, p$ defined by $q=\log k$ and $p=-\partial_{x} \log k-2 A_{0}$, the symplectic form is simply $\Omega=$ $\frac{1}{\lambda \lambda 2} \int_{0} \lambda 1 d x \delta q \delta p$. These Poisson brackets are those of the co-tangent bundle of the loop group $L \mathcal{R}$ of $\mathcal{R}$. In the next section, we will see how this may be understood using the Hamiltonian reduction approach.

Next we will compare the initial data of the Liouville theory with the parameters $k, A_{0}$ of the solutions of the theory. The Lagrangian symplectic form (3) parameterised in terms of the initial data $f(x)=\lambda \phi(x, 0)$ and $w(x)=\lambda \partial_{t} \phi(x, 0)$, is $\Omega=\frac{1}{\lambda \lambda 2} \int_{0} \lambda 1 d x \delta f \delta w$ and the associated Poisson brackets are $\{f(x), w(y)\}=$ $\lambda \lambda 2 \delta(x, y)$. The space of initial data with this symplectic structure is isomorphic to the Hamiltonian phase space $P_{H}$ of the Liouville theory and the initial data can be identified with the positions and momenta of the theory. Now we can easily express the initial data of the Liouville theory in terms of the parameters $k, A_{0}$. Indeed,

$$
(f, w)=\left(\log k,-\partial_{x} \log k-2 A_{0}\right)
$$

Since $f$ is a real function, the parameter $k$ is required to take values on the positive real line. Under this requirement, map given in eqn. (14) is a diffeomorphism, in 
fact a symplectic diffeomorphism, as the brackets (13) of $P_{C}$ become precisely the canonical brackets of $P_{H}$ under this map. Thus we conclude that the covariant $P_{C}$ and Hamiltonian $P_{H}$ phase spaces of the Liouville theory are isomorphic.

The solutions of the Liouville theory parameterised in terms of $A_{0}$ and $k$ ( $k>$ $0)$ are real. For solutions analytic in the time co-ordinate $t$, this can be shown explicitly by expressing these solutions as power series in $t$. For solutions which are not analytic in $t$, we note that the Liouville theory has a well-posed initial value problem [18], which amongst other things imples that there is a unique solution of the Liouville equation for every set of initial data $(f, w)$. Now we observe that if $\phi$ is a solution of the Liouville equation and $\phi$ is complex, then its complex conjugate $\phi \lambda *$ is a solution as well. However if the initial data $(f, w)(k>0)$ of $\phi$ are real, it is easy to prove that both $\phi$ and $\phi \lambda *$ have the same initial data. Hence they must be equal $(\phi=\phi \lambda *)$, and $\phi$ is real.

Finally, the solutions of the Liouville theory parameterised in terms of $A_{0}$ and $k$ can be re-written in terms of variables $n$ and $m$ such that $n$ depends on $x \lambda+$ and $m$ depends on $x \lambda$ - correspondingly. To do this, we choose a point $x_{0}$ on the real line and write the solution as $e \lambda \lambda \phi(x, t)=\epsilon_{i j} n \lambda i\left(x \lambda+, x_{0}\right) m \lambda j\left(x \lambda-, x_{0}\right)$ where $n\left(x, x_{0}\right)=W\left(a ; x_{0}, x\right) u_{0}$ and $m\left(x, x_{0}\right)=W\left(a ; x_{0}, x\right) v_{0}$. The solution $\phi$ does not depend on the choice of $x_{0}$.

\section{Hamiltonian Reduction}

An alternative approach to Liouville theory considers it as a Hamiltonian reduction of the Wess-Zumino-Witten (WZW) model with target space the group $S L(2, \mathcal{R})[19]$. We will now show that this reduction applies to the covariant canonical approach, in that the Hamiltonian reduction of the covariant canonical description of the WZW model, as given in ref. [16], yields the covariant canonical description of the Liouville theory presented above.

Firstly we recall some results from ref. [16]. The parameterisation of the classical solutions of the Lagrangian equations of motion of the WZW model on a 
cylinder with target space a compact and connected Lie group $G$ is

$$
\begin{aligned}
g(x, t) & =U(x \lambda+) \mathcal{W}(A ; x \lambda+, x \lambda-) V(x \lambda-), \\
\mathcal{W}(A ; x \lambda+, x \lambda-) & =P \exp \int_{x \lambda-} \lambda x \lambda+A(s) d s,
\end{aligned}
$$

where $U$ and $V$ are periodic maps from the real line $\mathcal{R}$ to the group $G$, and the field $A$ in the path-ordered exponential is a ( $\operatorname{Lie} G) \lambda *$-valued periodic one-form on the real line. The expression for $g(x, t)$ in eqn. (15) is then periodic in $x$ and solves the field equations

$$
\partial_{-}\left(\partial_{+} g g \lambda-1\right)=0,
$$

where $g$, a map from the cylinder into $G$, is the WZW field. The parameterisation (15) has the symmetry

$$
\begin{aligned}
& U(x) \rightarrow U(x) h(x), \quad V(x) \rightarrow h \lambda-1(x) V(x), \\
& A(x) \rightarrow-h \lambda-1(x) \partial_{x} h(x)+h \lambda-1(x) A(x) h(x),
\end{aligned}
$$

where $h$ is an element of the loop group of $G$. These results can be extended to include the WZW model whose target space is the group $S L(2, \mathcal{R})$.

Next we will show that a Hamiltonian reduction of this parameterisation of the space of solutions of the WZW model gives the parameterisation of the space of solutions of the Liouville field theory which we gave in the previous section.

The currents of the WZW model are given by

$$
J_{+}(x \lambda+)=-\frac{\kappa}{4 \pi} \partial_{+} g g \lambda-1, \quad J_{-}(x \lambda-)=\frac{\kappa}{4 \pi} g \lambda-1 \partial_{-} g .
$$

where $\kappa$ is a coupling constant. Hamiltonian reduction of the WZW model with target space the group $S L(2, \mathcal{R})$ to the Liouville theory occurs when one imposes 
the constraints

$$
\left.J_{+}\right|_{t \lambda-}=-\nu \frac{\kappa}{4 \pi},\left.\quad J_{-}\right|_{t \lambda+}=-\frac{\kappa \eta \lambda 2}{4 \pi \nu},
$$

on the components of the currents of the WZW model. Rewriting the constraints (16) in terms of the variables $U, V, A$ of the parameterisation (16), they become

$$
\begin{aligned}
& \left.\left(\partial_{+} U U \lambda-1+U A(x \lambda+) U \lambda-1\right)\right)\left.\right|_{t \lambda-}=\nu, \\
& \left.\left(V \lambda-1 \partial_{-} V-V \lambda-1 A(x \lambda-) V\right)\right|_{t \lambda+}=-\frac{\eta \lambda 2}{\nu} .
\end{aligned}
$$

Now we introduce $u \lambda i, v \lambda i$ satisfying the constraints (6). Then it is straightforward to show that the $S L(2, \mathcal{R})$ matrices

$$
U=\left(\begin{array}{cc}
\frac{u \lambda 1}{M} & \frac{u \lambda 2}{M} \\
-u \lambda 2 & u \lambda 1
\end{array}\right), \quad V=\left(\begin{array}{cc}
\frac{v \lambda 2}{N} & v \lambda 1 \\
-\frac{v \lambda 1}{N} & v \lambda 2
\end{array}\right)
$$

satisfy the constraints (20) where $M=(u \lambda 1) \lambda 2+(u \lambda 2) \lambda 2, N=(v \lambda 1) \lambda 2+(v \lambda 2) \lambda 2$. Notice that $M$ and $N$ cannot be zero for $u \lambda i, v \lambda i$ that satisfy the condition (6). Following the arguments of ref. [19], it then follows that the Liouville field $\phi$ is given by

$e \lambda \lambda \phi(x, t) \equiv(U W V) \lambda 2_{2}=(U(x \lambda+) W(A ; x \lambda+, x \lambda-) V(x \lambda-)) \lambda 2_{2}=\epsilon_{i j} u \lambda i(x \lambda+) W \lambda j_{l}(A ; x \lambda+, x \lambda-) v \lambda l$

where $(U W V) \lambda 2_{2}$ is the $(22)$ component of the $S L(2, \mathcal{R})$ matrix $U W V$. Thus, the Hamiltonian reduction defined by equation (19) reduces the WZW parameterisation (15) to the Liouville parameterisation (4). However, this Hamiltonian reduction does not necessarily lead to solutions $\phi$ of the Liouville theory that are real functions. The reality condition on these solutions arises as an additional restriction on their parameters and can be handled as in the previous section.

To show that the reduction is indeed Hamiltonian, i.e. that the WZW Poisson brackets reduce to the Liouville Poisson brackets, we recall that in ref. [16] we 
showed that the covariant canonical phase space of the WZW model was the cotangent bundle of the loop group of $G$, agreeing with the Hamiltonian phase space. The brackets are the canonical brackets on this phase space. This result also applies to the case $G=S L(2, \mathcal{R})$, and thus we have the result that the Poisson brackets of the WZW model with target space group $S L(2, \mathcal{R})$ in the gauge $U=1$ are

$$
\begin{aligned}
\{V(x), V(y)\} & =0 \\
\left\{V(x), A_{a}(y)\right\} & =\beta V(x) t_{a} \delta(x, y) \\
\left\{A_{a}(x), A_{b}(y)\right\} & =\beta\left(\delta_{a b} \frac{\partial}{\partial x} \delta(x, y)+f_{a b} \lambda c A_{c}(x) \delta(x, y)\right)
\end{aligned}
$$

where $V(x)$ is an element of $L S L(2, \mathcal{R})$ and $\beta=-\frac{4 \pi}{\kappa}$. The $t_{a}$ are the generators of $\operatorname{Lie}(S L(2, \mathcal{R})) \lambda *$, satisfying $\left[t_{a}, t_{b}\right]=f_{a b} \lambda c t_{c}$, with $f_{a b} \lambda c$ the structure constants of $S L(2, \mathcal{R})$. If we express $V$ in terms of $v$ as in eqn. (21), gauge fix $u$ and $v$ as in the previous section and use the algebra $[t \lambda \lambda 0, t \lambda \pm]= \pm 2 t \lambda \pm,[t \lambda+, t \lambda-]=t \lambda \lambda 0$ of the basis $t \lambda \lambda 0, t \lambda+, t \lambda-$, the Poisson brackets of eqn. (23) reduce to the Poisson brackets of eqn. (13) provided that $\beta=\frac{1}{2} \lambda \lambda 2$. Thus we see that the covariant canonical formulation of the Liouville theory presented in the previous section can be derived by a Hamiltonian reduction of the covariant canonical formulation of the WZW model which we gave in ref. [16].

\section{Conclusions}

The parameterisation described in section two can also be used to study the Liouville theory on a flat two-dimensional Minkowski space. The analysis for parameterising the solutions can be done in the same way as for the Liouville theory on the cylinder. However in this case, $u, v, A$ are not necesarily periodic. As a result the parameters $A_{0}$ and $k$ are smooth functions from the real line $\mathcal{R}$ into the real line $\mathcal{R}$. The calculation of the covariant symplectic form and the associated Poisson brackets can formally proceed as in the case of the Liouville theory on a cylinder. However, it should be noted that the covariant symplectic form is not well defined for all smooth $A_{0}, k, \delta A_{0}, \delta k$. 
To summarise, we have presented a parameterisation of the space of solutions of the Liouville theory on a cylinder with the following properties: The solutions of the Liouville theory are functions from a cylinder (space-time) into the real numbers. We showed that the space of parameters of the solutions is diffeomorphic to the space of initial data of the theory and that this diffeomorphism induces a symplectic diffeomorphism from the covariant phase space onto the Hamiltonian phase space of the Liouville theory. The Poisson brackets of the Liouville theory were also derived. Finally, we showed that our covariant phase space description of the Liouville theory can be also derived, via Hamiltonian reduction, from the covariant phase space description of the WZW model given by us in ref. [16].

Acknowledgements: G.P. was supported by the Commission of European Communities, and B.S. by a Queen Elizabeth II Fellowship from the Australian Government.

\section{REFERENCES}

1. J. Liouville, J. Math. Pure Appl. 18 (1853) 71.

2. A.M. Polyakov, Phys. Lett. B103 (1981) 207.

3. T.L. Curtright and C.B. Thorn, Phys. Rev. Lett. 48 (1982) 1309.

4. E. Braaten, T.L. Curtright and C.B. Thorn, Phys. Lett. 118B (1982) 115; Ann. Phys. 147 (1983) 365.

5. E. D'Hoker and R. Jackiw, Phys. Rev. D26 (1982) 3517.

6. E. Aldrovandi, L. Bonora, V. Bonservizi and R. Paunov, Free field representation of Toda field theories, SISSA-ISAS 210/92/EP.

7. J.-L Gervais and A. Neveu, Nucl. Phys. B238 (1984) 125; Nucl. Phys. B238 (1984) 396.

8. J.-L. Gervais, LPTENS preprint 92/36, hepth/9212109.

9. K. Gawedski, talk presented at the Oji Seminar on Quantum Analysis, Kyoto 1992, hepth/9210100. 
10. G. Weigt, Phys. Lett. B277 (1992) 79; DESY preprint 1992 (talk presented at Johns Hopkins Workshop).

11. O. Babelon, Phys. Lett. 215B (1988) 523.

12. O. Babelon, F. Toppan and L. Bonora, Commun. Maths. Phys. 140 (1991) 93

13. J.-L Gervais and A. Neveu, Nucl. Phys. B209 (1982) 125.

14. A.N. Leznov and M.V. Saveliev, Lett. Math. Phys. 3 (1979) 489.

15. V. Aldaya, J. Navarro-Salas and M. Navarro, CERN preprint TH. 6393/92, to appear in Contemporary Mathematics.

16. G. Papadopoulos and B. Spence, Phys. Lett. 295B (1992) 44.

17. G. Papadopoulos and B. Spence, The canonical structure of the WessZumino-Witten model, to appear in the Proceedings of the NATO ASI Conference "Low Dimensional Topology and Quantum Field Theory", Newton Institute for Mathematical Sciences, Cambridge, September 1992.

18. S.W. Hawking and G. F. R. Ellis, The large scale structure of space-time, CUP (1973).

19. P. Forgács, A. Wipf, J. Balog, L. Fehér and L. O’Raifeartaigh, Phys. Lett. 227B (1989) 214. 